\title{
X-linked adrenoleukodystrophy presenting as progressive ataxia and pure cerebellar involvement
}

Adrenoleucodistrofia ligada ao $\mathrm{X}$ apresentando-se como ataxia progressiva e envolvimento cerebelar isolado

Marianna Pinheiro Moraes de MORAES ${ }^{1}$, Augusto Bragança Reis ROSA ${ }^{1}$, Cristina Saade JAQUES ${ }^{1}$, Victor Hugo Rocha MARUSS1 ${ }^{2,3}$, José Luiz PEDROSO', Orlando Graziani BARSOTTINI ${ }^{1}$

A 27-year-old man presented with a two-year history of progressive ataxia. Family history was unremarkable. Examination revealed ataxia and alopecia. Serum cortisol levels were low, suggesting adrenal insufficiency. Brain magnetic resonance imaging (MRI) disclosed cerebellar white matter involvement (Figure 1). Exome sequencing showed homozygous mutations (c.268del p.Glu90Argfs*13) in the $A B C D 1$ gene and confirmed X-linked adrenoleukodystrophy (X-ALD).
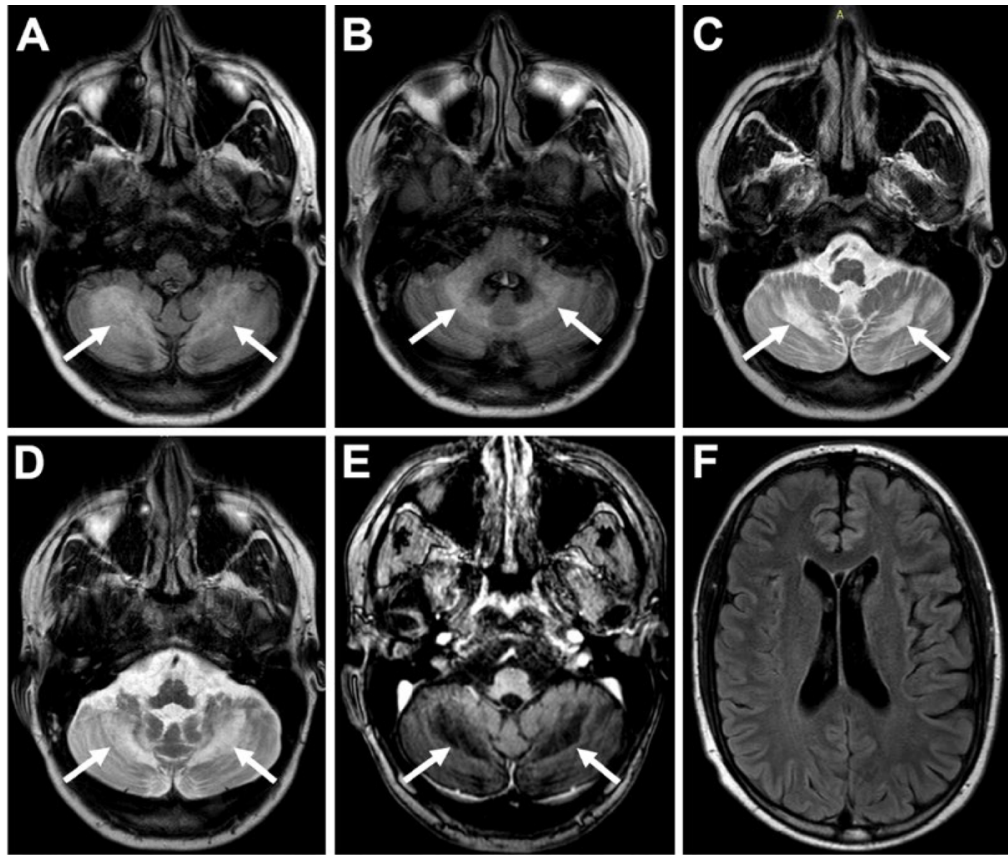

Figure 1. Patient with pure cerebellar ataxia related to X-linked adrenoleukodystrophy. Axial FLAIR-weighted brain MRI shows symmetrical cerebellar white matter and middle cerebellar peduncles hyperintense signal, besides pontocerebellar atrophy (A and B). Axial T2-weighted brain MRI discloses bilateral cerebellar white matter changes (C and D). Axial T1-weighted brain MRI reveals hypointense signal in cerebellar white matter (E). Axial FLAIR-weighted brain MRI shows no supratentorial abnormalities (F).

\footnotetext{
'Universidade Federal de São Paulo, Unidade de Ataxia, Departamento de Neurologia, São Paulo SP, Brazil. ${ }^{2}$ Hospital Beneficência Portuguesa de São Paulo, Departamento de Neurorradiologia, São Paulo SP, Brazil. ${ }^{3}$ Universidade Federal de São Paulo, Departamento de Radiologia, Divisão de Neurorradiologia, São Paulo SP, Brazil.

Marianna Pinheiro Moraes de MORAES (ID https://orcid.org/0000-0003-1273-9083; Augusto Bragança Reis ROSA (DD https://orcid.org/0000-0003-3447-7029; Cristina Saade JAQUES (D) https://orcid.org/0000-0002-2196-3151; Victor Hugo Rocha MARUSSI (D) https://orcid.org/0000-0002-0333-0342; José Luiz PEDROSO (D) https://orcid.org/0000-0002-1672-8894; Orlando Graziani BARSOTTINI (D) https://orcid.org/0000-0002-0107-0831

Correspondence: José Luiz Pedroso; E-mail: jlpedroso.neuro@gmail.com

Conflict of interest: There is no conflict of interest to declare.

Authors' contributions: Moraes MPM, Rosa ABR, Jaques CS, Marussi VHR, Pedroso JL and Barsottini OG: case report project: conception, organization, execution; manuscript: writing of the first draft, review and critique.

Received on June 22, 2020; Received in its final form on July 30, 2020; Accepted on September 19, 2020.
} 
Several forms of hereditary ataxias remain undetermined, despite being largely investigated. Whole-exome sequencing is a useful diagnostic approach for undetermined ataxias ${ }^{1}$. Adult-onset
X-ALD usually presents with behavioral changes, pyramidal signs, and white matter changes. Pure cerebellar white matter changes with progressive cerebellar ataxia are uncommon in X-ALD².

\section{References}

1. Németh AH, Kwasniewska AC, Lise S, Schnekenberg RP, Becker EBE, Bera KD, et al. Next generation sequencing for molecular diagnosis of neurological disorders using ataxias as a model. Brain. 2013 Oct;136(Pt 10):3106-18. https://doi.org/10.1093/brain/awt236
2. Chen YH, Lee YC, Tsai YS, Guo YC, Hsiao CT, Tsai PC, et al. Unmasking adrenoleukodystrophy in a cohort of cerebellar ataxia. PLoS One. 2017 May;12(5):e0177296. https://doi.org/10.1371/journal. pone. 0177296 